der Autor über die Suffixbildungen, über Lautanalogie und Volksetymologie. Alles in allem ist auch diese Arbeit Strömbergs außerordentlich verdienstlich und kenntnisreich.

B. Milt.

In Memoriam Prof. Dr. med. h. c. Dr. phil. Gustav Senn. Verh. Naturf. Ges. Basel 56, 2. Teil, 1945 Georg \& Co., Basel 1945, 560 S. mit Porträt und zahlr. Abbild.

Der als Festband für den 70. Geburtstag G. Senns (7. Nov. 1945) vorbereitete, von der Naturforschenden Gesellschaft Basel, der Schweiz. Gesellschaft für Geschichte der Medizin und der Naturwissenschaften u. a. herausgegebene stattliche Band wurde durch den am 10. Juli 1945 erfolgten Hinschied Senns zu einem posthumen Erinnerungsmal, errichtet von seinen Fachkollegen und Freunden.

Der reichbebilderte Band enthält neben 31 botanischen und vereinzelten physikalischen, mathematischen, geologischen, folkloristischen, philologischen, ethnographischen Aufsätzen - damit die weiten Interessen des Verstorbenen dokumentierend - auch sechs medizin- und naturwissenschaftshistorische Beiträge, die im folgenden kurz referiert sind. Nicht vergessen sei der dem Band vorangestellte biographische Abriß über Gustav Senn (1875-1945) aus der Feder Max Geiger-Huber's, der durch eine Liste der Veröffentlichungen Senns wertvoll ergänzt wird.

Die historischen Aufsätze leitet Hermann Geiger ein mit einem Beitrag über J. D. Labrams Insektenwerk (Seite 372). Die auch in bibliographischer Hinsicht verdienstliche Arbeit, welche in die sehr unübersichtlichen Druck- und Verlagsverhältnisse der von Labram illustrierten Insektenwerke Ordnung bringt, bildet eine willkommene Ergänzung zu Senn's Aufsätzen über Labrams Sammlung von Schweizer Pflanzen und von Giftpflanzen.

H. Fischer versucht in seinem Beitrag «Die Krankheitsauffassung Friedrich von Hardenbergs (Novalis) (1772-1801)» (S. 390) die spekulativ-philosophischen Grundlagen der Krankheitsauffassung Hardenbergs aus seiner Beschäftigung mit der zeitgenössischen Philosophie, Physik, Chemie usw. und aus der damals in Deutschland verbreiteten Cullen'schen und Brown'schen Medizin abzuleiten.

C. G. Jung analysiert in seinem Beitrag «Der philosophische Baum» (S. 411) den Baum als Symbol und Archetypus, welcher dem menschlichen Traumleben entstammt und zieht interessante historische Parallelen aus der hermetischen Literatur, wobei er auf die besondere Gestalt des «philosopischen Baumes» und seine uralten Zutaten: Schlange, Vögel usw. hinweist und seine symbolische Bedeutung als "Wandlungsform des Menschen» und als Symbol für das Mysterium von Leben, Tod und Wiedergeburt geistvoll interpretiert.

Charles Baehni macht in seiner interessanten Skizze: «Les idées de François Tingry dans l'œuvre de Charles Bonnet» (S. 424) auf die Bedeutung dieses 
Genfer Chemikers aufmerksam, welcher (mit den völlig unzulänglichen Mitteln seiner Zeit) eine chemische Analyse des Pollens und der weiblichen Fruchtanlage der Feuerlilie versuchte, die Charles Bonnet in seiner bekannten ovistischen Befruchtungstheorie verwertete. Ein neu aufgefundener Brief und ein Manuskript Tingrys bestätigen das Gesagte. Auf den Gegensatz BonnetGleditsch in der Befruchtungstheorie und die Verwertung der «chemischen» Resultate Tingrys zugunsten der Argumente Bonnets weist Baehni besonders hin.

R. von Fellenberg beleuchtet in seinem Beitrag «Die Neujahrsblätter der Gesellschaft zum Schwarzen Garten in Zürich (1808-1832)» (S. 446). Name, Herkunft, Bedeutung und Geschichte der «Gesellschaft» und bespricht die «neuen» Neujahrsblätter der Gesellschaft, welche sich mit den damals bekanntesten Badeorten der Schweiz, darunter Baden (1808), Pfäfers (1810), St. Moritz (1811), Schinznach (1813/15), Leuk (1816/17) u. a., aber auch mit solchen wie Stachelberg (1819), Nydelbad (1825), Gyrenbad (1826), Lostorf (1831) u. a. befassen, deren Ruhm im Laufe der Zeit etwas verblaßt ist.

Bernhard Peyer widmet in seinem Beitrag «Botanische Observationes aus den ersten Jahrzehnten der Ephemerides der Leopoldinischen Akademie» (S. 459) dem Jubilar einen reich illustrierten Blumenstrauß von Curiosa aus jener fruchtbaren Zeit, in welcher die reine Scheidung zwischen Phantasie, exakter Beobachtung und gedanklicher Analyse noch nicht überall vollzogen war. Damals war es noch erlaubt, mit wissenschaftlichem Ernst «Naturmythologeme» in wissenschaftlichen Zeitschriften zu publizieren und mit Entdeckerfreude über «Naturspiele» zu berichten.

Dieser kurzen Besprechung schließen wir ein Verzeichnis der biologiegeschichtlichen Arbeiten Gustav Senns an, welche wir dem von Max GeigerHuber mit Unterstützung von A. Becherer in Genf erstellten Gesamtverzeichnis seiner Arbeiten (Festband S. XI-XV) entnommen haben.

H. Fischer.

\section{Liste der Veröffentlichungen GUSTAV SENN's zur Geschichte der Naturwissenschaften, hauptsächlich der Antike}

1921 Die Pflanzenkunde des Theophrast von Eresos. - Sonntagsbl. d. Basler Nachrichten, 20. März 1921.

1922 Die Pflanzensystematik bei Theophrast von Eresos. - Verh. Schweiz. Naturf. Ges. Bern, 2. Teil, 302-303.

1923 Das pharmazeutisch-botanische Buch in Theophrast's Pflanzenkunde. Verh. Schweiz. Naturf. Ges. Zermatt, 2. Teil, 201-202.

1924 Weihrauch und Myrrhe. - «Die Ernte», 161-179 (und I-III). Friedrich Reinhard, Basel. 